Supplementary Material (cover)

\title{
Mapping cellular nanoscale viscoelasticity and relaxation times relevant to growth of living Arabidopsis thaliana plants using multifrequency AFM
}

\author{
Jacob Seifert ${ }^{1,2}$,Charlotte Kirchhelle ${ }^{2}$ Ian Moore ${ }^{2}$ and Sonia Contera ${ }^{1 *}$ \\ ${ }^{1}$ Clarendon Laboratory, Department of Physics, University of Oxford, Parks Road, Oxford OX1 3PU, UK \\ ${ }^{2}$ Department of Plant Sciences, University of Oxford, South Parks Road, Oxford OX1 3RB, UK \\ *corresponding author: sonia.antoranzcontera@physics.ox.ac.uk
}

\section{Contents:}

\section{Sections:}

S1. Mathematical model for quantitative mapping of viscoelastic properties of materials by multifrequency AFM: generalized Maxwell model.

S2. Hydrodynamic correction.

S3. Choosing the indentation model.

S4. Equations for mapping viscoelastic properties of materials that obey the MW and the SLS models using multifrequency AFM.

S5. Method used to choose the correct viscoelastic model: material dispersion graphs.

S6. Fitting equations for determining if a material obeys the SLS model using data from multifrequency AFM.

S7. Influence of the topography on the measurements, controls using plastic replica of the plants.

S8. Two dimensional AFM images and quantitative AFM mechanical maps.

S9. Growth rates determine the time interval of the experiments.

\section{Figures:}

S1. Comparison between photothermal and acoustic cantilever excitation in water.

S2. Behavior of amplitude and phase during an indentation.

S3. Justification of the indentation model.

S4. Half opening angle $\alpha$ of a Nanosensors PPP-NCAuLD tip.

S5. Processing flowchart including the experimental setup and calibration.

S6. Behavior of the scaling functions $\mathrm{w}_{1}$ and $\mathrm{w}_{2}$ versus $\omega \tau$.

S7. Comparison of the material dispersion graphs for the MW, SLS and KV models

S8. Comparison of the mechanical properties of an A. thaliana hypocotyl and a latex replica.

S9. 2D images of a 3 cell junction in the hypocotyl. (These images correspond to the data shown in the Fig. 2 of the main text).

S10. 2D images of a 3 cell junction in the hypocotyl in a different location from Fig. S10 and S11.

S11. 2D images of a 3 cell junction in the hypocotyl in a different location from Fig. S9 and S10.

S12. A. thaliana growth rates from $48 \mathrm{~h}$ to $60 \mathrm{~h}$ and $60 \mathrm{~h}$ to $72 \mathrm{~h}$ plotted against the four growth zones of the hypocotyl. 


\section{S1. Mathematical model for quantitative mapping of viscoelastic prop- erties of materials by multifrequency AFM: generalized Maxwell model}

The mechanical imaging technique used in this work is based on the method developed by Cartagena-Rivera et al. [1,2], which hereafter we will refer to as the "Cartagena-Rivera method". It is a contact resonance imaging method where the cantilever is permanently in contact with the sample, and the AFM imaging feedback is set on the deflection; simultaneously, the cantilever is driven at one or two oscillatory harmonics. Cartegena-Rivera et al. derived this method for the particular case of materials that can be described by the Kelvin-Voigt (KV) model, which is not a good description of the behavior of complex composite materials, as the theoretical analysis of stress-relaxation and creep experiments has shown [3]. The most general linear viscoelastic material can be described by the generalized Maxwell (MW) model, which accounts for the behavior of materials whose relaxation occurs on several timescales (Fig.1D of the main text). This section of the supplementary information (SI) will provide the derivation of new theory that extends the Cartagena-Rivera method to the case of general linear viscoelastic behavior, by linking the "observables" of a cantilever that is sinusoidally actuated, (namely the deflection $A_{0}$, the phase $\phi_{1}$ and the amplitude $A_{1}$ of the first harmonic oscillation), to the generalized MW model. The results of this derivation can be reduced to the KV, MW, and standardlinear solid (SLS), as we will be shown below. Finally, an approach to obtain the quantities $k_{i}$ (stiffness), $\tau_{i}$ (relaxation time), $\eta_{i}$ (viscosity) from the general 
MW model is presented.

To obtain a relation between the cantilever observables and the mechanical properties we will follow three steps: (i) The cantilever motion is described by a driven harmonic oscillator with damping to which an external force is applied. (ii) The external force will be determined by the material parameters, which implies that the force, exerted by an oscillating generalized MW material, will be determined. (iii) The cantilever motion will be linked to an appropriate indentation model (section S3).

The Cartagena-Rivera method uses the approximation of a driven harmonic oscillator moving in an external force field $F(t)$, following the equation:

$$
\frac{1}{\omega_{0}^{2}} \ddot{q}+\frac{1}{\omega_{0} Q} \dot{q}+q=\frac{F_{\mathrm{osc}} \sin (\omega t)}{k_{c}}+\frac{F(t)}{k_{c}} .
$$

The cantilever motion is characterized by its trajectory $q(t)$, resonance frequency $\omega_{0}$, spring constant $k_{c}$ and quality factor $Q$. The driving force oscillates with a force $F_{\text {osc }}$ and a frequency $\omega$. To solve this equation for a generalized MW material $F(t)$ must be the resulting force of such a material.

The steady state solution for $q(t)$ in eq. (S1) is given by the following expression:

$$
q(t)=A_{0}+A_{1} \sin \left(\omega t-\phi_{1}\right) .
$$

Every arm in the generalized MW material is characterized by its spring 
constant $k_{i}$ and its timescale $\tau_{i}=\eta_{i} / k_{i}$, with the viscosity $\eta_{i}$. Furthermore, in the generalized MW model the total strain $\delta$ must be the same as the strain $\delta_{i}, i=1 \ldots N$ in every arm, i.e. $\delta_{i}=\delta_{j} \equiv \delta, \forall i, j$. The total force $F$ is given by the sum of the forces $f_{i}$ in every arm and a relaxation, steady state, force $f_{\infty}$ by

$$
F=f_{\infty}+\sum_{i=1}^{N} f_{i}
$$

The force $f_{i}$ for the MW model is given by the partial differential equation (PDE) [3]

$$
k_{i} \frac{\partial \delta_{i}}{\partial t}=k_{i}\left(\frac{\partial \delta_{\text {dashpot }}}{\partial t}+\frac{\partial \delta_{\text {spring }}}{\partial t}\right)=\frac{1}{\tau_{i}} f_{i}+\frac{\partial f_{i}}{\partial t} .
$$

In the real space the PDEs cannot be simply summed but such an expression can be found in the Laplace domain, in which eq. (S4) has the form

$$
\hat{f}_{i}(s)=\frac{s k_{i}}{s+\frac{1}{\tau_{i}}} \hat{\delta}_{i}(s)
$$

The Laplace transform of a function $g(t)$ is denoted by $\hat{g}(s)=\mathcal{L}(g(t))(s)$, where $s$ is the free parameter in the Laplace domain. Because of the linearity of the Laplace transform, the total $F$ can be written in the Laplace domain as 


$$
\hat{F}(s)=\hat{f}_{\infty}+\sum_{i=1}^{N} \hat{f}_{i}(s)=\left(\frac{\hat{f}_{\infty}}{s}+\sum_{i=1}^{N} \frac{s k_{i}}{s+\frac{1}{\tau_{i}}}\right) \hat{\delta}(s) .
$$

The last equality is valid because $\delta_{i}=\delta, \forall i=1 \ldots N$. With the approximation of the cantilever motion as a driven harmonic oscillator, the material stimulation can be expected to be sinusoidal. This is the case in the steady state oscillation of the driven harmonic oscillator (eq. (S2)). Using these assumptions, the deformation $\delta(t)$ takes the form

$$
\delta(t)=\delta_{0}+A_{1} \sin \left(\omega t-\phi_{1}\right)
$$

In an atomic force microscopy (AFM) experiment the relation between $\delta(t)$ and $q(t)$ is given by

$$
\begin{gathered}
\delta(t)=-(Z+q(t)) \\
\delta_{0}=-\left(Z+A_{0}\right)
\end{gathered}
$$

where $Z$ is the position of the $z$-piezo.

The Laplace transform of eq. (S7) is 


$$
\hat{\delta}(s)=\frac{\delta_{0}}{s}+\frac{\omega}{s^{2}+\omega^{2}} A_{1} \cos \left(\phi_{1}\right)-\frac{s}{s^{2}+\omega^{2}} A_{1} \sin \left(\phi_{1}\right) .
$$

Substituting eq. (S9) into eq. (S5) and applying the inverse Laplace transform we obtain

$$
\begin{aligned}
f_{i}(t) & =\left(k_{i} \delta_{0}-A_{1} k_{i} \frac{1}{1+\omega^{2} \tau_{i}^{2}}\left(\omega \tau_{i} \cos \left(\phi_{1}\right)+\sin \left(\phi_{1}\right)\right)\right) e^{-\frac{t}{\tau_{i}}} \\
& +A_{1} k_{i} \frac{\omega^{2} \tau_{i}^{2}}{1+\omega^{2} \tau_{i}^{2}} \sin \left(\omega t-\phi_{1}\right) \\
& +A_{1} k_{i} \frac{\omega \tau_{i}}{1+\omega^{2} \tau_{i}^{2}} \cos \left(\omega t-\phi_{1}\right) .
\end{aligned}
$$

From eq. (S10) the initial modulus $\epsilon_{0, i}$, the storage modulus $\epsilon_{i}^{\prime}$, and the loss modulus $\epsilon_{i}^{\prime \prime}$ can be found [3]

$$
\begin{aligned}
\epsilon_{i, 0} & =k_{i}-\frac{1}{\delta_{0} \omega \tau_{i}}\left(\epsilon_{i}^{\prime} \cos \left(\phi_{1}\right)+\epsilon_{i}^{\prime \prime} \sin \left(\phi_{1}\right)\right) \\
\epsilon_{i}^{\prime} & =A_{1} k_{i} \frac{\omega^{2} \tau_{i}^{2}}{1+\omega^{2} \tau_{i}^{2}} \\
\epsilon_{i}^{\prime \prime} & =A_{1} k_{i} \frac{\omega \tau_{i}}{1+\omega^{2} \tau_{i}^{2}} .
\end{aligned}
$$

With eq. (S11) we can rewrite eq. (S10) as 


$$
\begin{gathered}
f_{i}(t)=\epsilon_{0, i} \delta_{0} e^{-\frac{t}{\tau_{i}}}+\epsilon_{i}^{\prime} A_{1} \sin \left(\omega t-\phi_{1}\right)+\epsilon_{i}^{\prime \prime} A_{1} \cos \left(\omega t-\phi_{1}\right) \\
\stackrel{\text { eq. (S7) }}{=} \epsilon_{0, i} \delta_{0} e^{-\frac{t}{\tau_{i}}}+\epsilon_{i}^{\prime}\left(\delta(t)-\delta_{0}\right)+\frac{\epsilon_{i}^{\prime \prime}}{\omega} \dot{\delta}(t) .
\end{gathered}
$$

Using both eqs. (S6) and (S10) and the linearity of the Laplace transform, the total force for the generalized MW model with a relaxation spring constant $k_{\infty}$ can be calculated

$$
\begin{aligned}
F(t) & =k_{\infty} \delta_{0}+k_{\infty}\left(\delta(t)-\delta_{0}\right)+\sum_{i=1}^{N}\left(\epsilon_{0, i} \delta_{0} e^{-\frac{t}{\tau_{i}}}+\epsilon_{i}^{\prime}\left(\delta(t)-\delta_{0}\right)+\frac{\epsilon_{i}^{\prime \prime}}{\omega} \dot{\delta}(t)\right) \\
& =\left(k_{\infty}+\sum_{i=1}^{N} \epsilon_{0, i} e^{-\frac{t}{\tau_{i}}}\right) \delta_{0}+\left(k_{\infty}+\sum_{i=1}^{N} \epsilon_{i}^{\prime}\right)\left(\delta(t)-\delta_{0}\right)+\left(\sum_{i=1}^{N} \epsilon_{i}^{\prime \prime}\right) \frac{\dot{\delta}(t)}{\omega} \\
& =E_{0}(t) \delta_{0}+E^{\prime}\left(\delta(t)-\delta_{0}\right)+E^{\prime \prime} \frac{\dot{\delta}(t)}{\omega} .
\end{aligned}
$$

In eq. (S13c) the total relaxation modulus $E_{0}$, storage modulus $E^{\prime}$ and loss modulus $E^{\prime \prime}$ are given by the following equations: 


$$
\begin{aligned}
E_{0}(t) & =k_{\infty}+\sum_{i=1}^{N} \epsilon_{0, i} e^{-\frac{t}{\tau_{i}}} \\
E^{\prime} & =k_{\infty}+\sum_{i=1}^{N} \epsilon_{i}^{\prime} \\
E^{\prime \prime} & =\sum_{i=1}^{N} \epsilon_{i}^{\prime \prime} .
\end{aligned}
$$

Equation (S14) is well known and implemented in finite element modelling (FEM) software packages, such as ABAQUS [4]. With the solution in eq. (S13) as external force in eq. (S1) we obtain

$$
\frac{1}{\omega_{0}^{2}} \ddot{q}+\frac{1}{\omega_{0} Q} \dot{q}+q=\frac{F_{\mathrm{osc}} \sin (\omega t)}{k_{c}}+\frac{E_{0}(t) \delta_{0}+E^{\prime}\left(\delta(t)-\delta_{0}\right)+E^{\prime \prime} \frac{\dot{\delta}(t)}{\omega}}{k_{c}} .
$$

The interaction will only happen when the cantilever is close to the surface, and therefore the quantities in eq. (S1) must be obtained near the surface, which will be denoted by the subscript 'near'. Substituting the steady state solution from eq. (S2) into eq. (S15), using the relations in eq. (S8) and adding in the driving force to the frequency the phase $\phi_{1}-\phi_{1}$, i.e. $F_{\text {drive }}=F_{\mathrm{osc}} \sin \left(\omega t+\phi_{1}-\phi_{1}\right)$, we obtain 


$$
\begin{array}{r}
-\frac{\omega^{2}}{\omega_{\text {near }}^{2}} A_{1} \sin \left(\omega t-\phi_{1}\right)+\frac{\omega}{Q_{\text {near }} \omega_{\text {near }}} A_{1} \cos \left(\omega t-\phi_{1}\right)+A_{1} \sin \left(\omega t-\phi_{1}\right)+A_{0} \\
=\frac{F_{\text {osc }}}{k_{c}}\left(\sin \left(\omega t-\phi_{1}\right) \cos \left(\phi_{1}\right)+\cos \left(\omega t-\phi_{1}\right) \sin \left(\phi_{1}\right)\right) \\
+\frac{1}{k_{c}}\left(E_{0}(t) \delta_{0}-E^{\prime} A_{1} \sin \left(\omega t-\phi_{1}\right)-E^{\prime \prime} A_{1} \cos \left(\omega t-\phi_{1}\right)\right) .
\end{array}
$$

Comparing now terms with $\sin \left(\omega t-\phi_{1}\right)$ and $\cos \left(\omega t-\phi_{1}\right)$ we get the relations between the observables $k_{c}, A_{1}, \phi_{1}$ and the material properties

$$
\begin{aligned}
k_{c} A_{0} & =E_{0}(t) \delta_{0} \\
E^{\prime} & =F_{\mathrm{osc}} \frac{\cos \left(\phi_{1}\right)}{A_{1}}-k_{c}\left(1-\frac{\omega^{2}}{\omega_{0}^{2}}\right) \\
E^{\prime \prime} & =F_{\mathrm{osc}} \frac{\sin \left(\phi_{1}\right)}{A_{1}}-k_{c} \frac{\omega}{Q \omega_{0}} .
\end{aligned}
$$

From Cartagena-Rivera et al.[1] we obtain the relations

$$
\begin{aligned}
F_{\mathrm{osc}} & =\frac{k_{c} A_{\mathrm{far}}}{Q_{\mathrm{far}}} \sqrt{1-\frac{1}{4 Q^{2}}} \\
1-\left(\frac{\omega}{\omega_{\text {near }}}\right)^{2} & =\frac{F_{\mathrm{osc}}}{k_{c}} \frac{\cos \left(\phi_{1, \text { near }}\right)}{A_{1, \text { near }}} \\
\frac{\omega}{\omega_{\text {near }} Q_{\text {near }}} & =\frac{F_{\text {osc }}}{k_{c}} \frac{\sin \left(\phi_{1, \text { near }}\right)}{A_{1, \text { near }}},
\end{aligned}
$$


which we can substitute into eq. (S16) and the final result is

$$
\begin{aligned}
F_{0}=k_{c} A_{0} & =E_{0}(t) \delta_{0} \\
E^{\prime} & =F_{\text {osc }}\left(\frac{\cos \left(\phi_{1}\right)}{A_{1}}-\frac{\cos \left(\phi_{1, \text { near }}\right)}{A_{1, \text { near }}}\right) \\
E^{\prime \prime} & =F_{\text {osc }}\left(\frac{\sin \left(\phi_{1}\right)}{A_{1}}-\frac{\sin \left(\phi_{1, \text { near }}\right)}{A_{1, \text { near }}}\right) .
\end{aligned}
$$

In our experiments, we measured the quantities $A_{1, \text { near }}$ and $\phi_{1, \text { near near the }}$ surface, at about $\sim 10 \mathrm{~nm}$ above the sample surface. This is needed to correct for hydrodynamic effects of the liquid [2, see section $\mathrm{S} 2$ for details]. $F_{0}$ is the force which is applied through the cantilever indentation and is measured by the cantilever spring constant and deflection $k_{c} A_{0}$. Equations (S23b) and (S23c) show the same relations between the cantilever observables and mechanical quantities as obtained by Cartagena-Rivera [1] but the quantities $E_{0}(t), E^{\prime}$, and $E^{\prime \prime}$ are different for the different models. The reason that the relations between the mechanical properties and the cantilever observables do not change, is that $E^{\prime}$ quantifies the stored energy in the system and is related to the displacement, while $E^{\prime \prime}$ is a measurement of the dissipated energy and is proportional to the velocity. Therefore, the left-hand-side (l.h.s.) in eq. (S23) will depend on the viscoelastic model applied to describe the material, whereas the right-hand-side (r.h.s.) is universal for all viscoelastic models.

For the SLS model $N=1$, the three moduli are given as 


$$
\begin{aligned}
E^{\prime} & =k_{\infty}+k_{1} \frac{\omega^{2} \tau_{1}^{2}}{1+\omega^{2} \tau_{1}^{2}} \\
E^{\prime \prime} & =k_{1} \frac{\omega \tau_{1}}{1+\omega^{2} \tau_{1}^{2}} \\
E_{0}(t) & =k_{\infty}+\left(k_{1}-\frac{A_{1}}{\delta_{0} \omega \tau_{1}}\left(\left(E^{\prime}-k_{\infty}\right) \cos \left(\phi_{1}\right)+E^{\prime \prime} \sin \left(\phi_{1}\right)\right)\right) e^{-\frac{t}{\tau_{1}}} .
\end{aligned}
$$

This is in agreement with the expected result [3].

The final contribution of the individual terms in eq. (S26) depends, also on the scan speed $v$ (although $v$ is not directly involved in eq. (S26), it determines the time $t$ the cantilever spends at a pixel), the initial indentation $\delta_{0}$, and the oscillation amplitude $A_{1}$. Usually the term with $A_{1} / \delta_{0} \omega \tau_{1}$ is negligible, because the assumption for the derivation was $A_{1} \ll \delta_{0}$ and for a polymer we expect that $\omega \tau_{1} \sim \mathcal{O}(1)$, where $\mathcal{O}()$ is the Landau symbol, i.e. $\omega \tau_{1}$ is of the order of 1. Furthermore, $v$ is low enough so that the time spent at a pixel $t_{p} \gg \tau_{1}$ and therefore the material is considered to be in the relaxed state and we obtain

$$
E_{0}(t) \approx k_{\infty}
$$

\section{S2. Hydrodynamic correction}

As demonstrated in the previous section, it is important for quantitative dynamic measurements in liquids to correct for the hydrodynamic coupling between cantilever and surface. To keep this coupling as weak as possible we used 


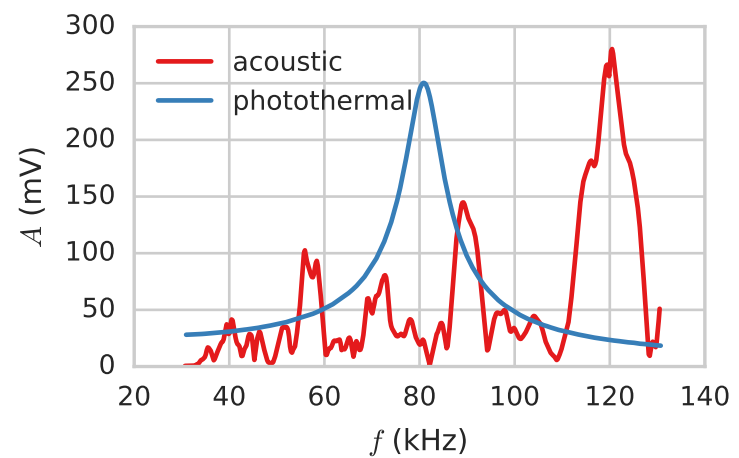

Figure S1: Comparison between photothermal and acoustic cantilever excitation in water. The plot shows amplitude vs frequency behavior of the cantilever used in the experiments for acoustic (red) and photothermal actuation (blue). The advantage of photothermal excitation in water is that the resonance of the cantilever can be described as a harmonic oscillator and depends only weakly on the distance of the cantilever to the surface (distance dependence not shown). The multiple peaks in the case of acoustic excitation originate from the force of a water wave interacting with the cantilever. The water wave is caused by the oscillation of the cantilever chip.

photothermal excitation of the cantilever. Unlike acoustic excitation, which drives the whole chip of the cantilever, photothermal excitation drives only the cantilever (Fig. S1) and consequently the excitation of the water is reduced by the ratio of the surface area of the two. This ensures that the cantilever response is linear. This simple response allows a correction for the hydrodynamic coupling. It should be mentioned here that any other direct excitation method of the cantilever (e.g. magnetic excitation or Lorentz force excitation) would be valid for the application of this technique. However, the Lorentz force excitation that we used in our previous work [5] requires special cantilevers, which are only produced in limited designs. Due to the complex topology of plant tissues, high aspect ratio cantilevers are required to avoid contact between the cantilever and the cells. Such cantilevers are not commercially available for 


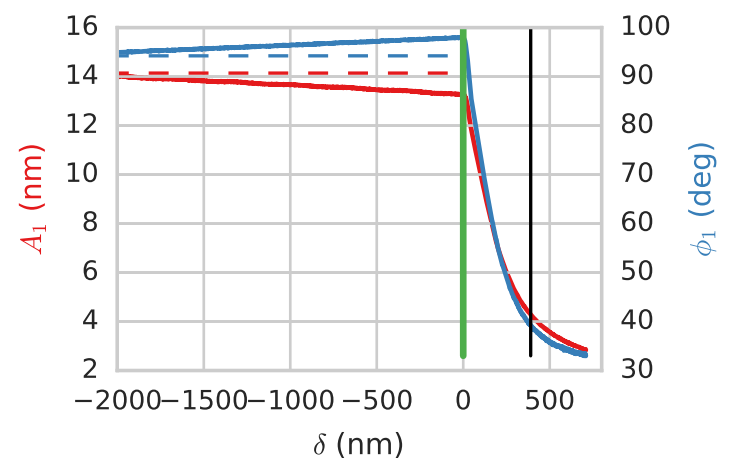

Figure S2: Behavior of amplitude and phase during indentation. The graphs show the behaviou of amplitude $A_{1}$ and phase $\phi_{1}$ during an indentation experiment. The green line shows the contact point with the sample and the dashed/solid lines show $A_{1}$ and $\phi_{1}$ far away from the surface and close to the surface, respectively.

Lorentz force excitation.

Having a system were a hydrodynamic correction can be applied in a simple way, makes this an easy procedure. An amplitude-distance curve and a phasedistance curve are measured while the cantilever is oscillating at the desired frequency; from these curves $A_{1}$ and $\phi_{1}$ just above the surface can be calculated (Fig. S2). Usually, we chose a distance about $10 \mathrm{~nm}$ above the surface to obtain those values. It must be noted that the tissue topography can influence the hydrodynamic coupling. This means the hydrodynamic coupling will increase, as the area of the cantilever close to the sample increases (due to the shape of the sample).To obtain more accurate results, additional measurements of $A_{1}$ and $\phi_{1}$ above the sample were performed before each scan.

It is possible to obtain the error of the measurement as follows. Using the equations S23: 


\section{S3. Choosing the indentation model}

To link the viscoelastic values of the SLS model which was used in the derivation above, to 3D dimensional moduli an appropriate indentation model has to be used. This is not straightforward since the plant cell wall is a complex composite material. However, we can make two assumptions that simplify the problem:(i) at small scales (such as the size of the volumes at which the indentation takes place) the sample behaves isotropically. And (ii) the cell wall is much thicker than the oscillatory indentation depth, which is about $\sim 5 \mathrm{~nm}$. With these conditions we can assume that the major contribution to the measurement comes from indenting a thick material rather than bending it and we can use indentation theory. For linear elasticity we can use the Hertz model and we have the following relationship between the force $F$ and indentation $\delta$

$$
F=g \frac{E}{1-\nu^{2}} \delta^{\gamma},
$$

where $\nu$ is the Poisson ratio of the sample, $E$ the Young's modulus, and $g$ and $\gamma$ are geometrical coefficients specific to the indenter geometry.

In the case of the Sneddon model (shown in Fig. S3) $g=2 \tan (\alpha) / \pi$ with $\alpha \approx 15^{\circ}$ being the half opening angle, which is given by the manufacturer of the cantilever[6] (shown in Fig. S4) and $\gamma=2$ [7].

From this model the three dimensional (3D) moduli can be determined following the approach of Raman et al. [5] and Cartagena-Rivera et al. [1]. With 

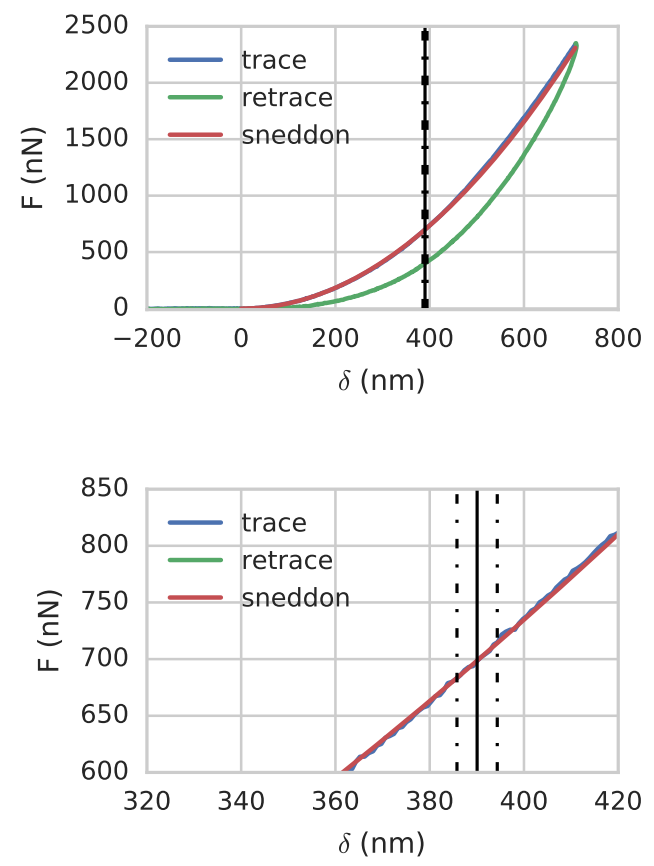

Figure S3: The Sneddon model is a good fit for the indentation curve. The red curve in the top panel shows the fit to the loading indentation curve (trace, blue); the unloading curve (retrace) is displayed in green. The solid black line shows the imaging indentation depth and the dashed line show the oscillation amplitude during imaging. The lower panel shows an expansion of the panel, to magnify the fit curve around the area that corresponds to the conditions of the AFM imaging.

a Taylor series expansion of the indentation model in eq. (S28) we obtain

$$
\begin{aligned}
F(\delta) & =F\left(\delta_{0}\right)+\frac{\partial F}{\partial \delta}+\frac{\partial F}{\partial(\omega \delta)} \dot{\delta} \\
& =g \frac{E_{0}(t)}{1-\nu^{2}} \delta_{0}^{\gamma}+\gamma g \frac{E_{3 D}^{\prime}}{1-\nu^{2}} \delta_{0}^{\gamma-1}\left(\delta-\delta_{0}\right)+\gamma g \frac{E_{3 D}^{\prime \prime}}{1-\nu^{2}} \delta_{0}^{\gamma-1} \frac{\dot{\delta}}{\omega}
\end{aligned}
$$

The introduction of $E_{3 D}^{\prime}$ and $E_{3 D}^{\prime \prime}$ is justified by the information we deduced from the one dimensional (1D) derivation, namely that $E^{\prime}$ is related to the 


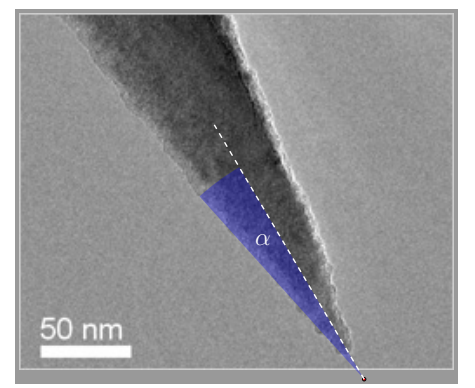

Figure S4: Half opening angle $\alpha$ of a Nanosensors PPP-NCAuLD tip. The angle has to be measured for a virtual tip, which is outside the real probe, since the real tip size is finite.

displacement $\left(\delta(t)-\delta_{0}\right)$ and $E^{\prime \prime}$ is related to the velocity $\dot{\delta}(t)$ (eq. (S13)).

Comparing eq. (S30) with eq. (S13) we find

$$
\begin{aligned}
F\left(\delta_{0}\right) & =F_{0}=g \frac{E_{0}(t)}{1-\nu^{2}} \delta_{0}^{\gamma} \\
E_{1 D}^{\prime} & =\gamma g \frac{E_{3 D}^{\prime}}{1-\nu^{2}} \delta_{0}^{\gamma-1} \\
E_{1 D}^{\prime \prime} & =\gamma g \frac{E_{3 D}^{\prime \prime}}{1-\nu^{2}} \delta_{0}^{\gamma-1}
\end{aligned}
$$

From here the three 3D moduli can be obtained from the following expressions 


$$
\begin{aligned}
\delta_{0} & =\sqrt[\gamma]{\frac{F_{0}}{E_{0}} \frac{1-\nu^{2}}{g}} \\
E_{3 D}^{\prime} & =\frac{E_{1 D}^{\prime}}{\gamma} \sqrt[\gamma]{\frac{1-\nu^{2}}{g}} \sqrt[\gamma]{\frac{E_{0}}{F_{0}}} \gamma-1 \\
E_{3 D}^{\prime \prime} & =\frac{E_{1 D}^{\prime \prime}}{\gamma} \sqrt[\gamma]{\frac{1-\nu^{2}}{g}}{\sqrt[\gamma]{\frac{E_{0}}{F_{0}}}}^{\gamma-1} .
\end{aligned}
$$

$E_{0}$ can be determined from the quasi-static indentation as shown in fig. S3 and with the assumption of eq. (S27), $E_{0}$ is the relaxation modulus, which we assume to be constant for the whole sample. We then obtain $F_{0}, E_{1 D}^{\prime}, E_{1 D}^{\prime \prime}$ from the AFM scan. Furthermore, we assume the cell wall is an incompressible material and therefore $\nu=0.5$. Fig. S3 demonstrates that our indentation is well described by the Sneddon model, i.e. $\gamma=2, g=\frac{2}{\pi} \tan (\alpha)$, and also that the experiment was done in the limit of small oscillations where the linear approximation fits well (lower panel). In these conditions we obtain from eq. (S32)

$$
\begin{aligned}
E_{3 D}^{\prime} & =\frac{E_{1 D}^{\prime}}{2} \sqrt{\frac{1-\nu^{2}}{\tan (\alpha)} \frac{\pi}{2} \frac{E_{0}}{F_{0}}} \\
E_{3 D}^{\prime \prime} & =\frac{E_{1 D}^{\prime \prime}}{2} \sqrt{\frac{1-\nu^{2}}{\tan (\alpha)} \frac{\pi}{2} \frac{E_{0}}{F_{0}}}
\end{aligned}
$$

It is important to highlight, that Cartagena-Rivera [1] chose $E_{3 D}^{\prime}$ instead 
of $E_{0}$ because they used the $\mathrm{KV}$ model. This approach overestimates the material stiffness because it neglects the relaxation which happens throughout the scan in contact mode. Comparing the mathematical expressions from eq. (S33) with the result previously obtained by Cartagena-Rivera [1] $E_{3 D}^{\prime}=$ $\pi\left(1-\nu^{2}\right){E^{\prime}}_{1 D}^{2} /\left(8 \tan (\alpha) F_{0}\right)$, it can be shown that using the $\mathrm{KV}$ mode, the overestimation of the $\mathrm{KV}$ is given by a factor

$$
\frac{E_{3 D}^{\prime \mathrm{GMW}}}{E^{\prime \mathrm{KV}} \mathrm{V}_{3 D}}=\sqrt{\frac{1-\nu^{2}}{\tan (\alpha)}} \frac{\pi}{2} \frac{E_{1 D}^{\prime}}{\sqrt{F_{0} E_{0}}} \approx 1.3 \frac{E_{1 D}^{\prime}}{\sqrt{F_{0} E_{0}}} .
$$

The same relation can be found for $E^{\prime \prime}$. With a relaxation modulus of $E_{0} \approx 30 \mathrm{MPa}$ and an applied force of $F_{0} \approx 750 \mathrm{nN}$ and $E_{1 D}^{\prime} \approx 40 \mathrm{~N} \mathrm{~m}^{-1}$ the $\mathrm{KV}$ model provides values which are about a factor 4.25 larger than the generalized MW model.

To summarize, in order to obtain dynamic viscoelastic maps the following conditions and experimental order must be fulfilled:

- $A_{0} \gg A_{1}$ to ensure linearization of the problem, i.e. contact area does not change significantly during oscillation (see also fig. S3).

- The experiment must take place in the repulsive regime, i.e. $\phi_{1}<90^{\circ}$

- Force-volume curves must be obtained after every scan and record the parameters $A_{0}, A_{1}, \phi_{1}$ (and if measured in the experiment, also observables for higher harmonics). The calibration quantities near the surface should be measured at about $10 \mathrm{~nm}$ above the surface and the quantities far from 


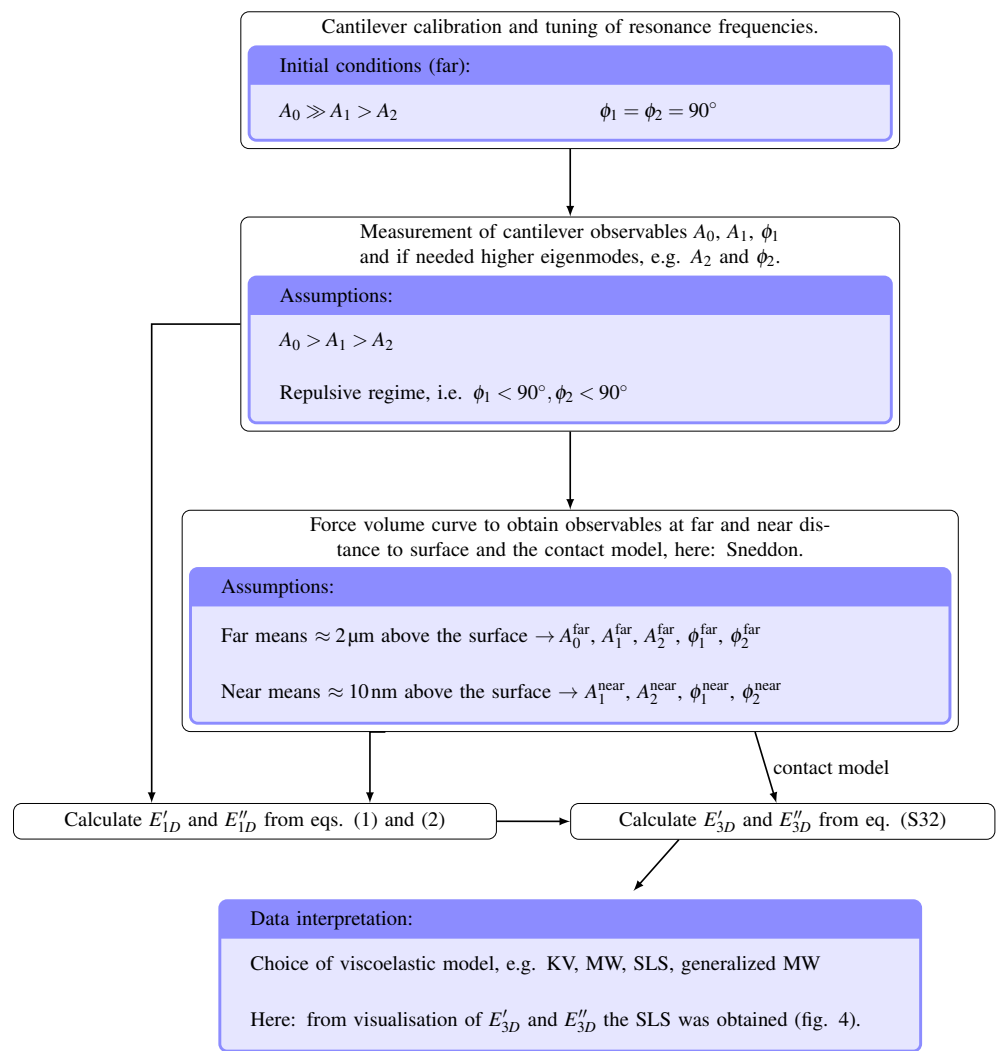

Figure S5: Processing flowchart including the experimental setup and calibration, and summary of assumptions necessary for calculating $E^{\prime}$ and $E^{\prime \prime}$.

the surface should be measured at least $2 \mu \mathrm{m}$ above the surface. This curve is also important to determine the contact model, i.e. exponent $\gamma$ in eq. (S28).

- With the scan and the calibration quantities $E_{1 D}^{\prime}$ and $E_{1 D}^{\prime \prime}$ can be calculated.

- With the contact model and the fitted Young's modulus $E_{0}$ from this model and the one dimensionalmquantities $E_{3 D}^{\prime}$ and $E_{3 D}^{\prime \prime}$ can be determined. 
- The choice of the viscoelastic model, e.g. MW, KV, SLS, is important for the data interpretation but not for the experiment.

enditemize

The whole process, including mathematical and experimental conditions is summarized in a flowchart (??).

S4. Equations for mapping viscoelastic properties of materials that obey the MW and the SLS models using multifrequency AFM

From eqs. (S24) and (S25) we obtain

$$
\frac{E^{\prime}}{E^{\prime \prime}}=\frac{\kappa}{\omega \tau}+(1+\kappa) \omega \tau \equiv \Theta(\kappa, \omega \tau) .
$$

We call $\Theta(\kappa, \omega \tau)$ the time response function; we introduced $\kappa=k_{\infty} / k_{\mathrm{M}}$. For the MW model we obtain the relations for $k_{\mathrm{M}}$ and $\eta$

$$
\begin{gathered}
k_{\mathrm{M}}=\frac{{E^{\prime}}^{2}+E^{\prime \prime 2}}{E^{\prime}} \\
\eta=\frac{1}{\omega} \frac{E^{\prime 2}+E^{\prime \prime 2}}{E^{\prime \prime}} .
\end{gathered}
$$


Using the r.h.s. of eq. (S35) we obtain for the SLS model

$$
\begin{aligned}
\frac{E^{\prime 2}+E^{\prime \prime 2}}{E^{\prime}} & =E^{\prime}+\frac{E^{\prime \prime}}{\Theta(\kappa, \omega \tau)} \\
& =k_{0}-k_{\mathrm{M}}+k_{\mathrm{M}} \frac{\omega^{2} \tau^{2}}{1+\omega^{2} \tau^{2}}+k_{\mathrm{M}} \frac{\omega \tau}{1+\omega^{2} \tau^{2}} \frac{1}{\Theta(\kappa, \omega \tau)} \\
& =k_{0}-k_{\mathrm{M}}\left(1-\frac{\omega^{2} \tau^{2}}{1+\omega^{2} \tau^{2}}\left(1+\frac{1}{\Theta(\kappa, \omega \tau) \omega \tau}\right)\right) \\
& \equiv k_{0}-k_{\mathrm{M}}\left(1-w_{1}(\kappa, \omega \tau)\right) \\
\frac{1}{\omega} \frac{E^{\prime 2}+E^{\prime \prime 2}}{E^{\prime}} & =E^{\prime \prime}\left(1+\left(\frac{E^{\prime \prime}}{E^{\prime}}\right)^{2}\right) \\
& =\eta \frac{1+\Theta(\kappa, \omega \tau)^{2}}{1+\omega^{2} \tau^{2}} \equiv \eta w(\kappa, \omega \tau)
\end{aligned}
$$

where we introduced the weight factors $w_{1}(\kappa, \omega \tau), w_{2}(\kappa, \omega \tau)$

$$
\begin{aligned}
& w_{1}(\kappa, \omega \tau)=\frac{\omega^{2} \tau^{2}}{1+\omega^{2} \tau^{2}}\left(1+\frac{1}{\Theta(\kappa, \omega \tau) \omega \tau}\right) \\
& w_{2}(\kappa, \omega \tau)=\frac{1+\Theta(\kappa, \omega \tau)^{2}}{1+\omega^{2} \tau^{2}} .
\end{aligned}
$$

Both $w_{1}$ and $w_{2}$ depend on the significant timescale $\omega \tau$ but $w_{1} \in[0,1]$ introduces a reduction of $k_{0}=k_{\infty}+k_{\mathrm{M}}$ by a percentage of $k_{\mathrm{M}}$, whereas $w_{2} \geq 1$ introduces an additional resistance which arises from the presence of $k_{\infty}$, which can be seen by considering the equality $w_{2}=1 \Leftrightarrow \kappa=0 \Rightarrow$ 


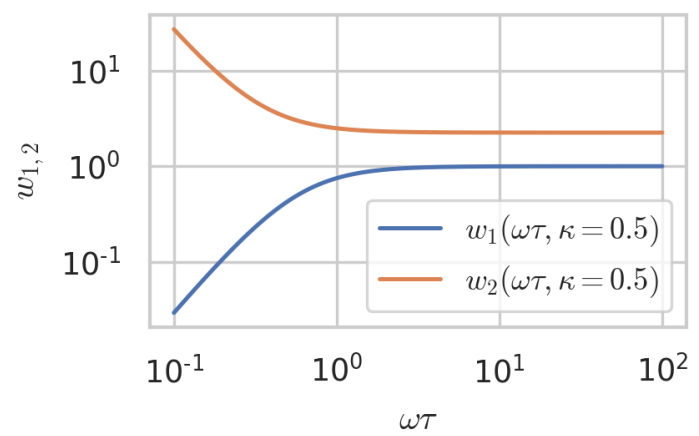

Figure S6: Behavior of the scaling functions $w_{1}$ and $w_{2}$ versus $\omega \tau$. We note that scaling functions do not have units.

$$
k_{\infty}=0 .
$$

S5. Method used to choose the correct viscoelastic model: material dispersion graphs

In order to choose the viscoelastic model that better describes the mechanical behavior of the material studied by the multifrequency AFM technique used in this paper, we plot $E^{\prime}$ and $E^{\prime \prime}$ against the inverse loss tangent $E^{\prime} / E^{\prime \prime}$, i.e. we use the material dispersion graphs. The theory developed in previous sections shows that the graphs for each model have specific functions that can be fitted for experimental data. In Fig. S7 we show the theoretical predictions for the SLS, MW and KV models. 

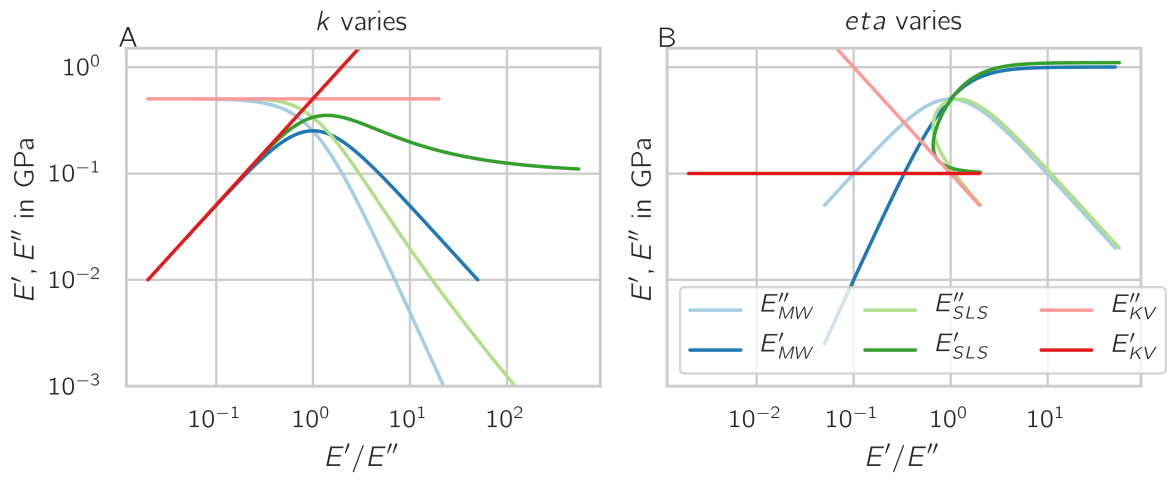

Figure S7: Comparison of the material dispersion graphs for the MW (blue), SLS (green) and KV (red) models (a), only $k$ changes (as in the main text) and (b) when only $\eta$ changes

S6. Fitting equations for determining if a material obeys the

\section{SLS model using data from multifrequency AFM}

To fit the data presented in Fig. 3(B) in the main text to the functions plotted in Fig. S7, it is necessary to find a representation of the moduli, which depends on the ratios $R=E^{\prime} / E^{\prime \prime}$ and the relevant timescale $\omega \tau$. Using eqs. (S24) and (S25) one can obtain the following relationships

$$
\begin{aligned}
& E^{\prime}\left(R, k_{\infty}, \omega \tau\right)=k_{\infty}\left(1+\frac{\omega \tau}{R-\omega \tau}\right) \\
& E^{\prime \prime}\left(R, k_{\infty}, \omega \tau\right)=\frac{k_{\infty}}{R-\omega \tau}
\end{aligned}
$$

With $R$ given from the experiment, the function $\left\|g\left(E^{\prime}, E^{\prime \prime}\right)-f\left(E^{\prime}, E^{\prime \prime}\right)\right\|^{2}$ with $g\left(E^{\prime}, E^{\prime \prime}\right)$ being the data and $f\left(E^{\prime}, E^{\prime \prime}\right)$ given by 


$$
f\left(R, p_{0}, p_{1}\right)=\left(\begin{array}{c}
E^{\prime}\left(R, p_{0}, p_{1}\right) \\
E^{\prime \prime}\left(R, p_{0}, p_{1}\right)
\end{array}\right)
$$

where $E^{\prime}$ and $E^{\prime \prime}$ given by

$$
\begin{aligned}
& E^{\prime}\left(R, p_{0}, p_{1}\right)=p_{0}\left(1+\frac{\omega \tau}{R-p_{1}}\right) \\
& E^{\prime \prime}\left(R, p_{0}, p_{1}\right)=\frac{p_{0}}{R-p_{1}}
\end{aligned}
$$

can be minimized. As initial parameters for the $p_{0}=\operatorname{mean}\left(E^{\prime}\right)$ and $p_{1}=\operatorname{mean}(R)-5 * \operatorname{std}(R)$ where used. The mean and the standard deviation std are calculated using all the pixels corresponding to each cell wall.

\section{S7. Influence of the topography on the measurements, controls using plastic replica of the plants}

A frequently discussed issue is whether the topography of the cells influences the mechanical properties. This hypothesis was tested using a latex replica of the hypocotyl. If the characteristic distribution in the mechanical mapping in Fig. S8 (a) was caused by the topography, the mechanical profile of the replica Fig. S8 (c) should qualitatively have the same distribution. However, the results of Fig. S8 clearly indicate that this is not 

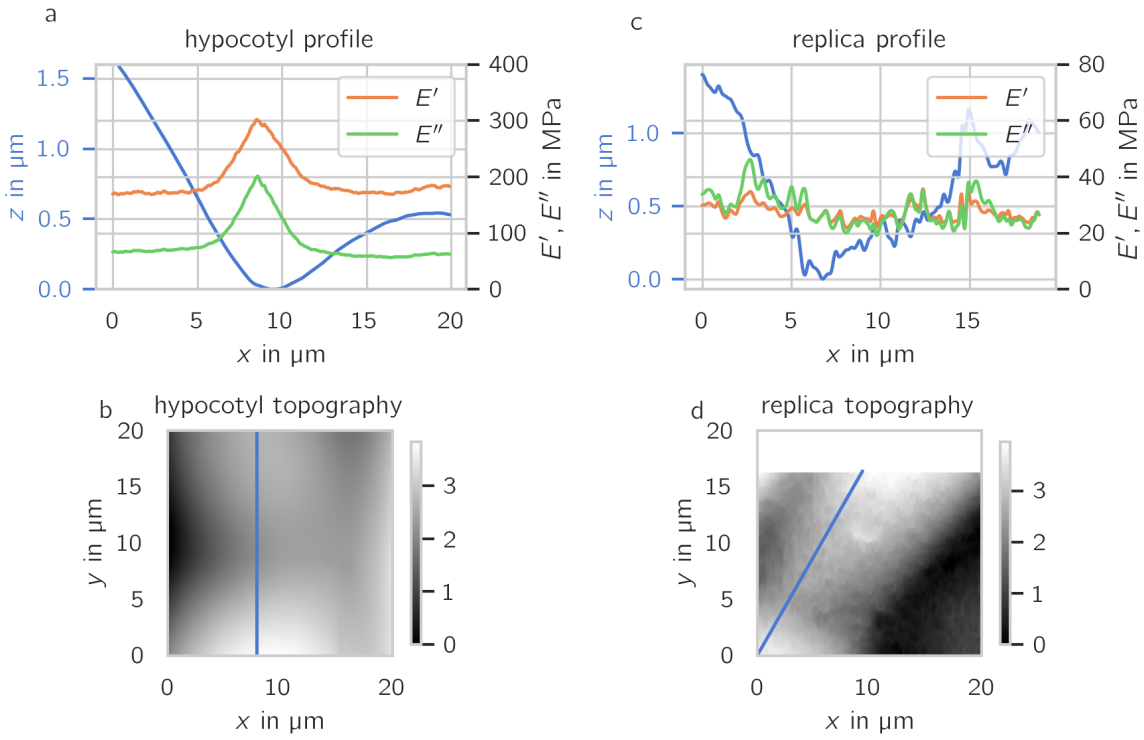

Figure S8: Comparison of the mechanical properties of an A. thaliana hypocotyl and a latex replica of an $A$. thaliana hypocotyl. The line profile of the topography of the seedling and the corresponding $E^{\prime}$ and $E^{\prime \prime}$ values along the line are shown in (a). These values correspond to the profile marked with a blue line in the topographic image in (b).(c) Shows the line profile of the topography of the replica, which is indicated with a blue line in (d), and the corresponding values of $E^{\prime}$ and $E^{\prime \prime}$ along the line.

the case. These results also demonstrate that the hydrodynamic effect is not responsible for the mechanical properties contrast at the cell junction measured in our experiments, but it is indeed caused by differences in the mechanical properties of the cell wall.

\section{S7.1 Experimental preparation of hypocotyl casts and moulds}

To investigate topographical contributions during mechanical mapping it was useful to create replicas of the sample to use as a control, made from less complex materials in order to asses the contribution of the topography alone. Existing protocols [8] turned out to not being useful for our viscoelasatic mapping, because the dental waxes used in those studies are 
too stiff for the investigation of dynamic mechanical properties. Instead we used a polymorphic plastic compound (Thermomorph) to create the mould.

The plastic was warmed in hot water to $70^{\circ} \mathrm{C}$ until it become soft enough to be deformed easily by the seedlings. For a high quality cast the plastic was spread evenly on a substrate. It was important to achieve a smooth surface, while at the same time, the compression of the material should not create domains in deeper layers because this causes a resistance when the seedling was pressed into the material. To avoid these artefacts the plastic was formed into a small ball of a diameter $d \approx 5 \mathrm{~mm}$. The plastic ball was then compressed between two glass slides into a thin layer. Then the top slide was removed and the seedling was gently pressed onto the plastic layer using a glass slide. Once the material became rigid, both the slides and the seedling were removed. During the whole process it was useful to keep a hydration layer on the plastic. This helped when removing the slides and the seedling (which are both hydrophilic whereas the plastic is hydrophobic).

The casts were filled with liquid moulding latex from Vesey (Birmingham, UK). The amount required depended on the size of the seedling. Before the mould could be attached to the sample holder, unnecessary parts were removed with a razor blade. This made it easier to find the replica on the latex surface. The mould can be attached with any adhesive to the holder. We used the medical adhesive. 
S8. Two dimensional AFM images and quantitative AFM mechanical maps

In this section we show AFM images of $A_{0}, F_{0}, A_{1}, \phi_{1}$, which are the observables used to calculate the quantitative maps of $E^{\prime}$ and $E^{\prime \prime}$. Fig. S9 corresponds to the images displayed in Fig. 2 of the main text. Two additional locations in the hypocotyl are displayed in Figs. S10 and S11. 
a

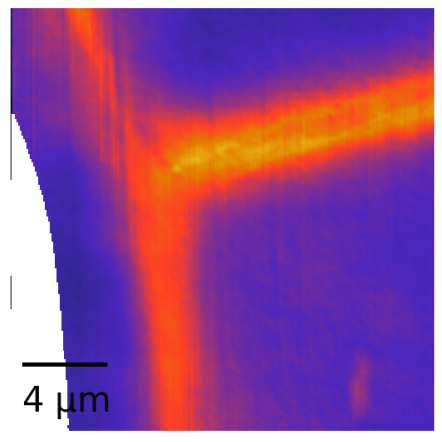

C

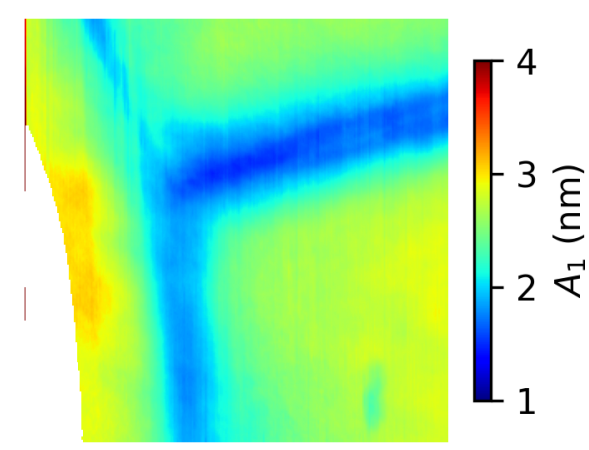

e

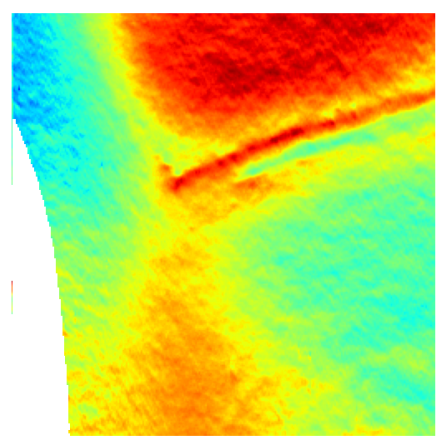

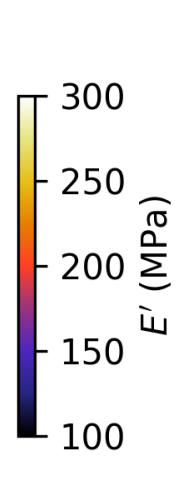

b

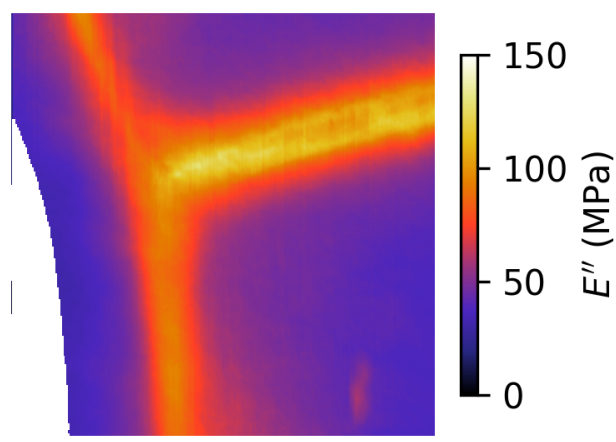

d

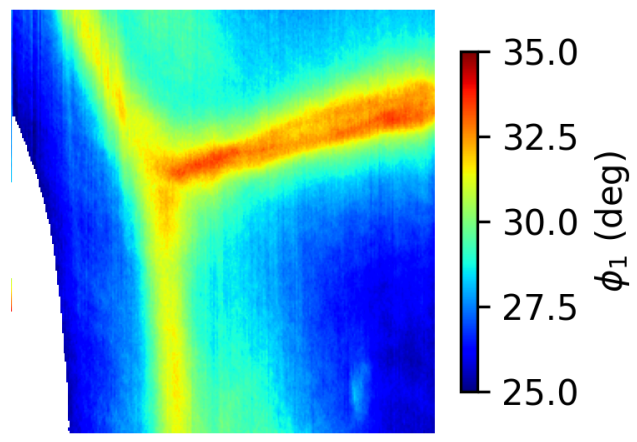

f

20.0

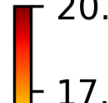

17.5

15.0 气

$-12.5$

10.0

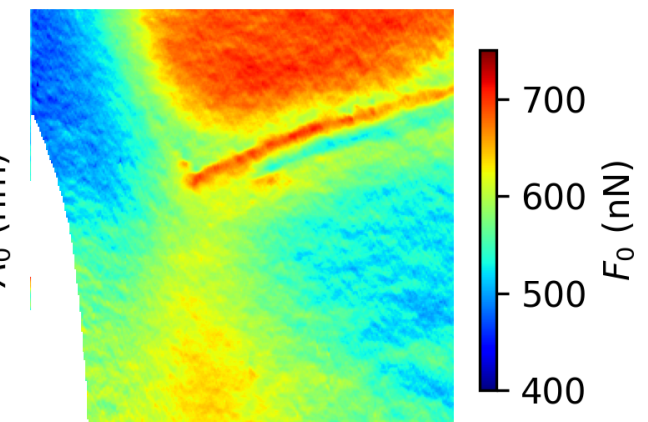

Figure S9: 2D images of a 3 cell junction in the hypocotyl including maps of (a) $E^{\prime}$, (b) $E^{\prime \prime}$, (c) $A_{1}$, (d) $\phi_{1}$, (e) $A_{0}$, and (d) $F_{0}$. These images correspond to the data shown in the Fig. 2 of the main text. 
a
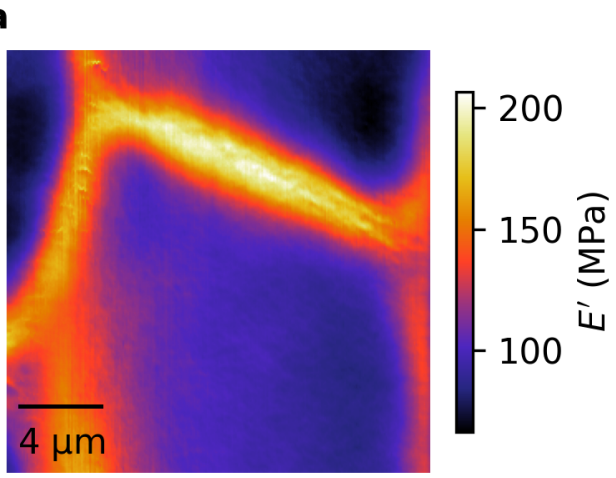

C

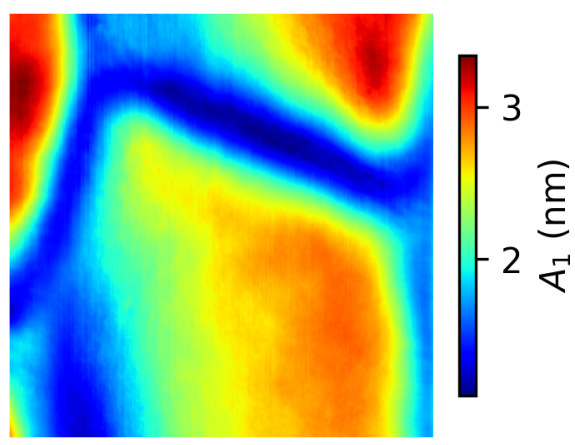

e

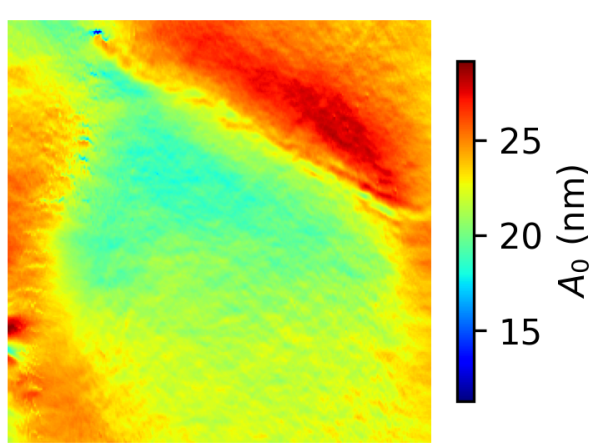

b

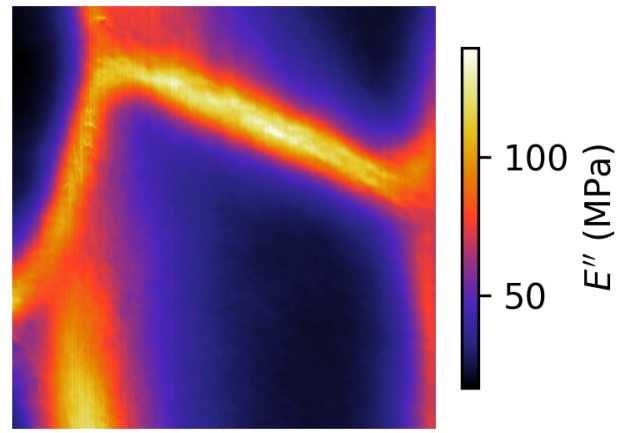

d

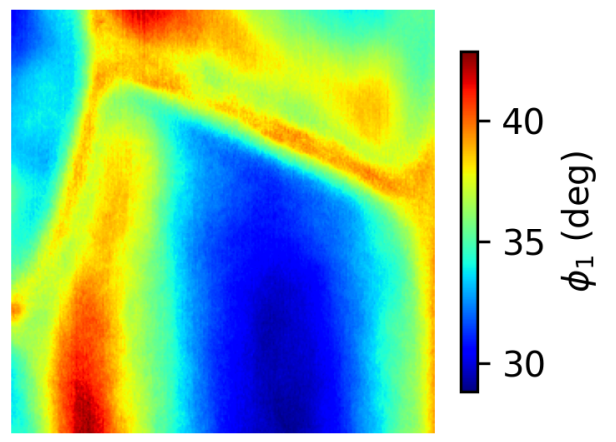

$\mathbf{f}$

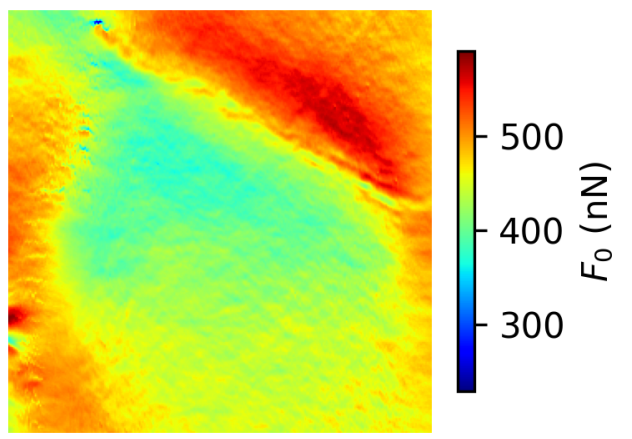

Figure S10: 2D images of a 3 cell junction in the hypocotyl in a different location from Fig. S9 and S1 1 , including maps of (a) $E^{\prime}$, (b) $E^{\prime \prime}$, (c) $A_{1}$, (d) $\phi_{1}$, (e) $A_{0}$, and (d) $F_{0}$. 
a
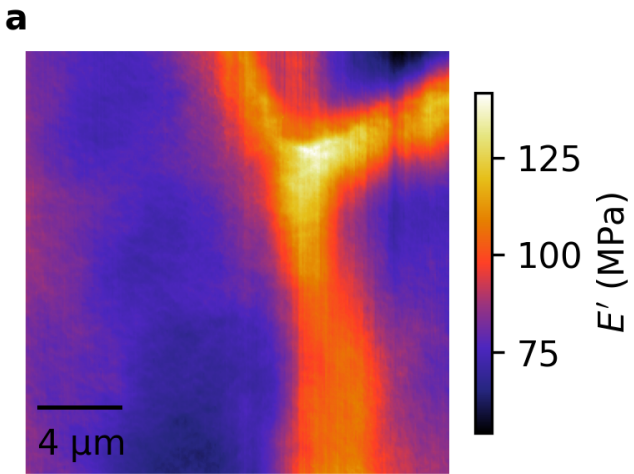

C
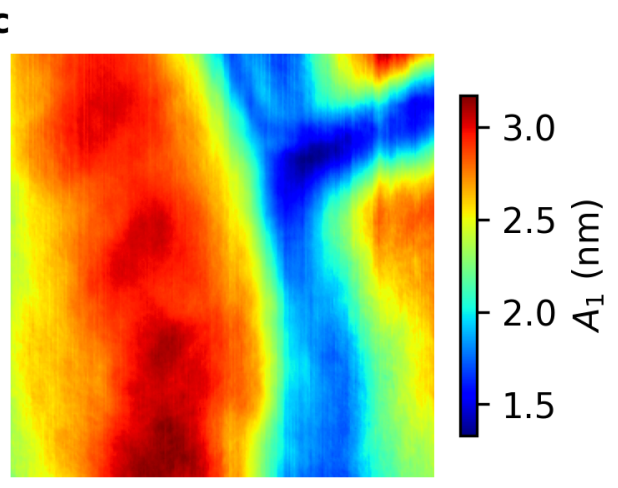

e

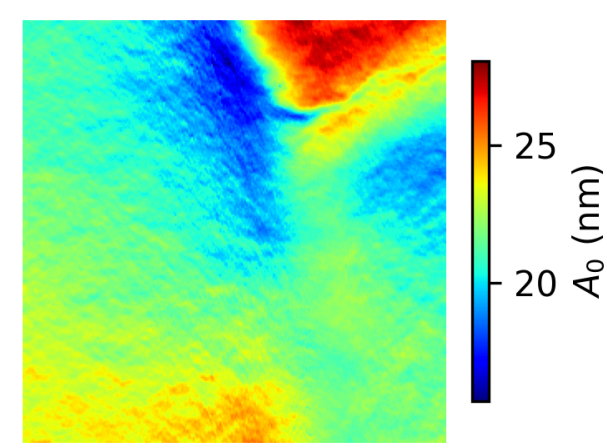

b

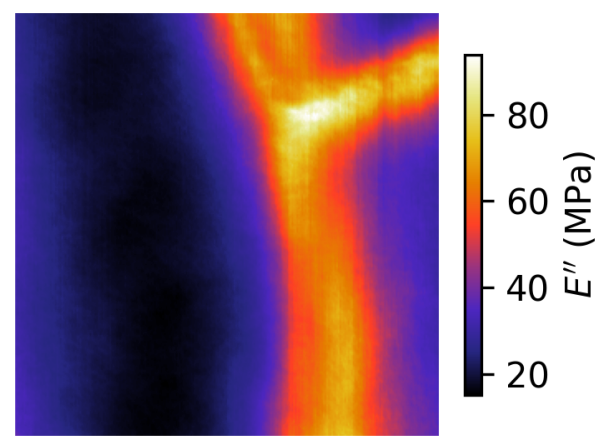

d

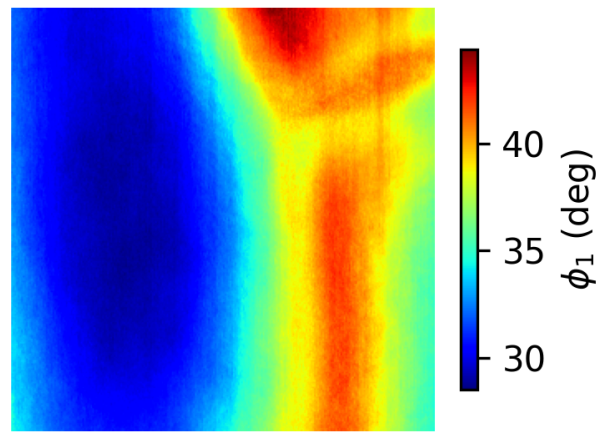

f

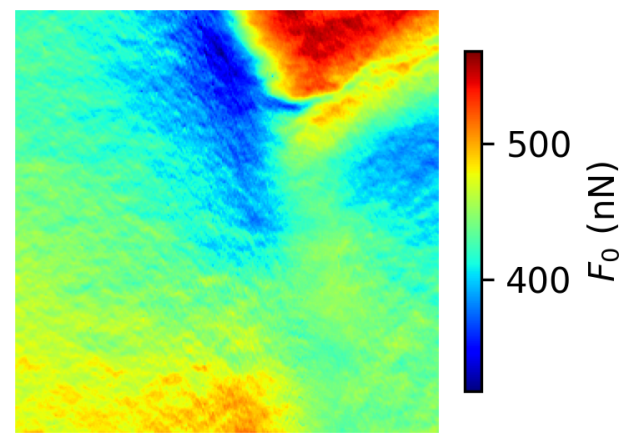

Figure S1 1: 2D images of a 3 cell junction in the hypocotyl in a different location from Fig. S9 and S10, including maps of (a) $E^{\prime}$, (b) $E^{\prime \prime}$, (c) $A_{1}$, (d) $\phi_{1}$, (e) $A_{0}$, and (d) $F_{0}$. 


\section{S9. Growth rates determine the time interval of the experiments}

For determining the correlation of mechanical properties with growth rates, we chose to perform experiments on dark-grown hypocotyls, which are known to display a developmental gradient along their longitudinal axis [9]. Basal cells expand first, with a wave of growth subsequently shifting in acropetal direction. To quantify cellular growth rates in different regions of the hypocotyl under our growth conditions, we examined growth rates in two time intervals: $48 \mathrm{~h}$ to $60 \mathrm{~h}$ and $60 \mathrm{~h}$ to $72 \mathrm{~h}$ after shifting plants to the growth chamber. Figure S12 shows the average hourly growth rate between $48 \mathrm{~h}$ to $60 \mathrm{~h}$ and $60 \mathrm{~h}$ to $72 \mathrm{~h}$ old seedlings after germination. It can be clearly seen the two intervals display a significantly different growth behavior recapitulating the acropetal wave of growth described in the literature. In the first time interval, cells at the base displayed the most rapid growth, whereas this growth peak had shifted towards zone 3 in the second time interval. We performed our AFM experiments at $60 \mathrm{~h}$ to capture actively growing cells at different regions 1 to 4 . Based on the assumption that cell wall mechanical properties undergo significant changes to drive the observed shift in growth rate along the hypocotyl in the time scales observed here, we subsequently focused on a narrower time interval $(58 \mathrm{~h}$ to $62 \mathrm{~h})$ to quantify growth rates relevant to our AFM mechanical measurements. 


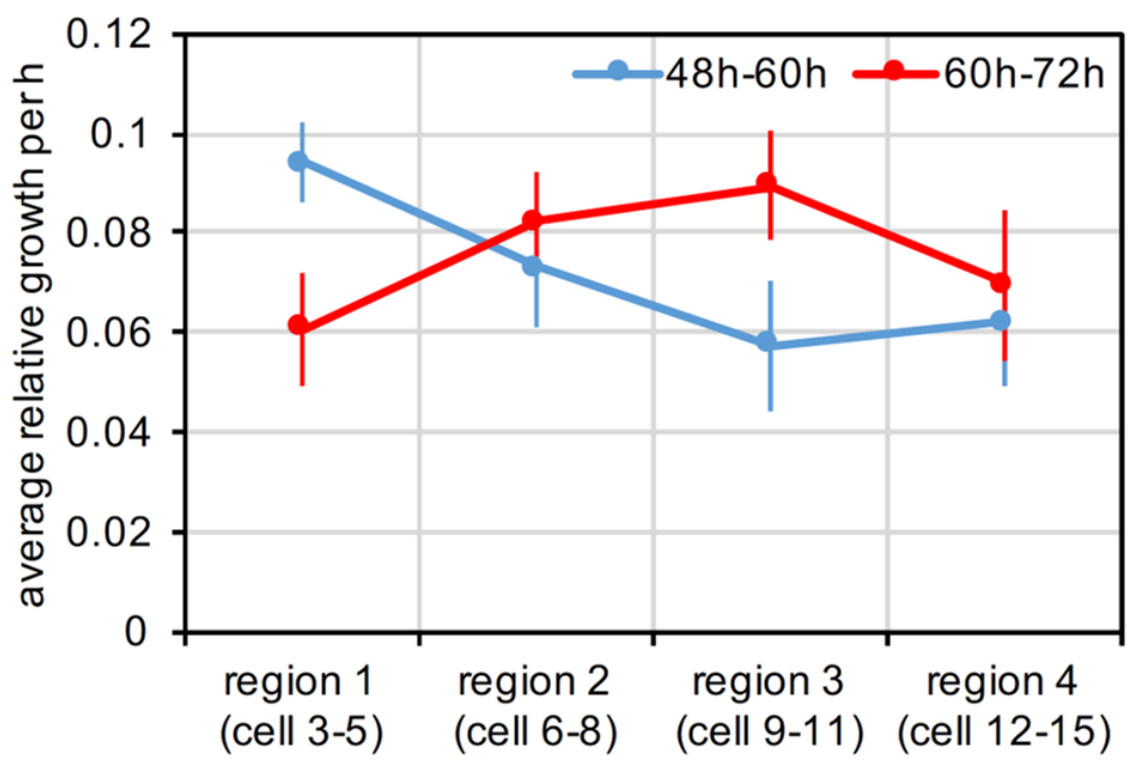

Figure S12: A. thaliana growth rates from $48 \mathrm{~h}$ to $60 \mathrm{~h}$ and $60 \mathrm{~h}$ to $72 \mathrm{~h}$ plotted against the four growth zones of the hypocotyl. The error bars represent standard deviation.

\section{References}

[1] Alexander X. Cartagena-Rivera, Wen-Horng Wang, Robert L. Geahlen, and Arvind Raman. Fast, multi-frequency, and quantitative nanomechanical mapping of live cells using the atomic force microscope. Scientific Reports, $5: 11692,2015$.

[2] Alexander Cartagena and Arvind Raman. Local viscoelastic properties of live cells investigated using dynamic and quasi-static atomic force microscopy methods. Biophysical Journal, 106(5):1033-1043, 2014.

[3] M. Doi. Soft Matter Physics. Oxford University Press, 2013.

[4] M. Smith. Abacus standard user's manual. Dassault Systemes Simulia Corporation, 6.9, 2009. 
[5] A. Raman, S. Trigueros, A. Cartagena, A. P. Z. Stevenson, M. Susilo, E. Nauman, and S. Antoranz Contera. Mapping nanomechanical properties of live cells using multi-harmonic atomic force microscopy. Nature Nanotechnology, 6(12):809-814, 2011.

[6] Nanosensors. https://www.nanosensors.com/pointprobe-plus-non-contacttapping-mode-long-cantilever-au-coating-detector-side-afm-tip-ppp-nclaud. 2020.

[7] I.N. Sneddon. The relation between load and penetration in the axisymmetric boussinesq problem for a punch of arbitrary profile. Journal of Engineering Science, 3:47-57, 1965.

[8] J. Dumais and D. Kwiatkowska. Analysis of surface growth in shoot apices. Plant Journal, 31(2):229-241, 2002.

[9] E. Gendreau, J. Traas, T. Desnos, O. Grandjean, M. Caboche, and H. Hofte. Cellular basis of hypocotyl growth in arabidopsis thaliana. Plant Physiology, 114(1):295-305, 1997. 